\title{
Cultura ciudadana para el manejo de residuos sólidos mediante la investigación como estrategia pedagógica
}

\section{Citizen culture for solid waste management through research as a pedagogical strategy}

DOI: http://dx.doi.org/10.17981/cultedusoc.9.3.2018.17

Artículo de investigación. Fecha de recepción: 15/06/2018. Fecha de aceptación: 27/11/2018

Rosa Córdoba-Meriñón

Iluminada Cantillo-Manjarrez; Marilis De Horta-Martínez; Eleida Guerra-Arocha; María Monsalve-Muñoz; José Sánchez-Medina; Mariela Rada-Brieva;

María Correa-Torres; Carlos Alcalá-Sierra; César Silva-Barrios; Luz García-Passo; Esmeralda Granados-Rada; Héctor Jiménez-Oliveros; Alex Meléndez-García; Victoriana Orozco-Sánchez; Carlos Rodríguez-Rayo y Dora Dennys-Saavedra ${ }^{2}$

Para citar este artículo:

Córdoba-Meriño, R., Cantillo-Manjarrez, I., De Horta-Martínez, M., Guerra-Arocha, E., Monsalve-Muñoz, M., Sánchez-Medina, J., Rada-Brieva, M., Correa-Torres, M., Alcalá-Sierra, C., Silva-Barrios, C., García-Passo, L., Granados-Rada, E., Jiménez-Oliveros, H., Meléndez-García, A., Orozco-Sánchez, V., Rodríguez-Rayo, C. y Dennys-Saavedra, D. (2018). Cultura ciudadana para el manejo de residuos sólidos mediante la investigación como estrategia pedagógica. Cultura. Educación y Sociedad 9(3), 141-152. DOI: http://dx.doi. org/10.17981/cultedusoc.9.3.2018.17

\section{Resumen}

A nivel mundial, en América latina y el Caribe, la cultura ciudadana para el manejo de residuos sólidos es una debilidad a nivel poblacional y cultural que termina contaminando el medio. El estudio tuvo como propósito fomentar la cultura ciudadana para el manejo de residuos sólidos mediante la investigación como estrategia pedagógica. La metodología se orientó bajo el enfoque cualitativo, utilizando el tipo de investigación acción, desde un diseño descriptivo, exploratorio. Para la recolección de datos se utilizaron técnicas como; observación participante y diario de campo. La población estuvo conformada por sesenta (60) estudiantes de la IED Tercera Mixta del municipio de Fundación Magdalena. Dando como resultado el diseño de actividades pedagógicas para identificar las concepciones y hábitos de los estudiantes con respecto al manejo de los residuos sólidos generados en la institución propiciando conductas pro ambientales como reciclaje, reutilización y reducción de residuos sólidos generados en la institución y sus alrededores.

Palabras clave: cultura ciudadana, residuos sólidos, investigación como estrategia pedagógica.

\section{Abstract}

At the global level, in Latin America and the Caribbean, the citizen culture for solid waste management is a weakness at the population and cultural level that ends up contaminating the environment. The purpose of the study was to promote citizen culture for solid waste management through research as a pedagogical strategy. The methodology was oriented under the qualitative approach, using the type of action research, from a descriptive, exploratory design. For the collection of data, techniques such as; participant observation and field diary. The population was conformed by sixty (60) students of the Third Mixed IED of the municipality of Magdalena Foundation. Resulting in the design of pedagogical activities to identify the conceptions and habits of students regarding the management of solid waste generated in the institution, promoting pro environmental behaviors such as recycling, reuse and reduction of solid waste generated in the institution and its surroundings.

Keywords: Education, research, ICT, citizen culture and solid waste.

1 Líder del grupo de investigación "Proteccion Ambiental Terceriana".

2 Docentes IED Tercera Mixta pertenecientes al Grupo de investigación "Citeeins".

- The author; licensee Universidad de la Costa - CUC Cultura, Educación y Sociedad vol. 9 no. 3, pp. 141-152. Diciembre, 2018 Barranquilla. ISSN 2389-7724 Online 


\section{Introducción}

En Colombia, al igual que muchos países en el mundo, enfrenta grandes retos en el manejo integral de sus residuos sólidos municipales (RSM). Esto debido en gran parte, y principalmente, al elevado índice de crecimiento demográfico e industrial del país, al cambio de hábitos de consumo de la población, la elevación de los niveles de bienestar, y la tendencia a abandonar las zonas rurales para concentrarse en los centros urbanos.

Existen graves daños provocados al ambiente por el manejo inadecuado de los RSM, entendiendo manejo como las diferentes fases del ciclo de vida de los residuos desde que se generan, almacenan, transportan, tratan y disponen en algún sitio. Tal situación se debe a que, por mucho tiempo en Colombia, en algunas regiones y sobre todo el departamento del Magdalena, el control sobre los RSM ha sido inadecuado y aún no se logra la incorporación de técnicas modernas de administración para la solución de este problema que, en forma directamente proporcional al tiempo que pasa, se va agravando. Los esfuerzos que realizan los gobiernos municipales, estatales y nacional, así como los demás sectores de la sociedad, no han estado siempre lo suficientemente concatenados para alcanzar resultados tangibles respecto a la solución del reto que representa la gestión integral de los residuos sólidos municipales.

En la actualidad se vive una transformación radical a través de las coordenadas del conocimiento, la cultura, la sociedad y la tecnología que es necesario contextualizar para construir condiciones de convivencia y mejor calidad de vida para los ciudadanos (Arrieta y Rojas, 2002). Así, la cultura ciudadana se expresa a través de las formas de organización y de convivencia social y de la manera de transformar los recursos naturales para la satisfacción de las necesidades e intereses humanos (Alaniz, 2016). En este sentido, las sociedades del mundo deben apuntar a formar para la ciudadana como una opción fundamental que "genere sentido de pertenencia, facilite la convivencia y conduzca al respeto del patrimonio común y al reconocimiento de los derechos y deberes ciudadanos" (Alcaldía de Bogotá, 1995).

En Colombia, formar para la ciudadanía debe ser una tarea urgente y de suma importancia, ya que en el país se viven diariamente situaciones que vulneran los indicadores antes mencionados, demostrando la carencia de formación en cultura ciudadana, es así como en el Plan de desarrollo de Colombia al año 2019 se ha formulado la visión de promover ciudadanos libres y responsables, cuya finalidad es la construcción de ciudadanía; una ciudadanía con sentido de pertenencia, tolerante, capaz de concertar y cumplir acuerdos, y sobre todo, una ciudadanía responsable, que respete la ley y cumpla las normas de convivencia por voluntad propia y no solo por imposición legal.

No obstante, las sociedades no han sido capaces de generar condiciones que garanticen los comportamientos del ser humano en beneficio de la construcción de una mejor sociedad. Formar para la ciudadanía es sin dudad una tarea difícil, pues, las sociedades viven en permanente conflicto, la inseguridad aqueja a las comunidades, la corrupción es generalizada y la falta de conciencia ambiental está acabando con el planeta, pues durante muchas décadas, el hombre, a través de sus prácticas cotidianas, ya sean de tipo doméstico o industrial, ha generado una diversidad de materiales que son considerados de desecho ejerciendo un manejo inadecuado sobre estos.

Dadas las condiciones que anteceden, se considera que elevar el nivel de cultura ambiental de la población es una prioridad, y es únicamente a través de la educación como el individuo interioriza la cultura, y 
es capaz de construir y producir conocimientos, reorientar sus valores, modificar sus acciones y contribuir como sujeto individual a la transformación de la realidad del medio ambiente_(Alaniz, 2016).

Es conveniente señalar, que el espacio geográfico donde se desarrolla la propuesta de investigación que se describe el este informe, es un municipio ubicado en el departamento del Magdalena, también conocido como la esquina del progreso, una población que cuenta aproximadamente con una población de 82.532 habitantes de los cuales el $90 \%$ se encuentra en el casco urbano y el $10 \%$ restante corresponde a los habitantes del área rural, los cuales están distribuidos así: Santa Rosa, Santa Clara, Resguardo Indígena y Doña María. La ubicación geográfica del municipio ha propiciado convertirse en el gran punto comercial de la zona, pues en él confluyen los habitantes de otros municipios y corregimientos aledaños (Aracataca, Retén, Pivijay, entre otros), para desarrollar sus actividades comerciales, lo cual origina que a diario se de gran actividad y movimiento de diferentes culturas que dejan su evidencia en el rastro que queda luego de esa interacción que genera la dinámica comercial.

Asimismo, su población se caracteriza por su gran calor humano, por su alegría, sencillez y empuje para enfrentar las situaciones que impiden el desarrollo del municipio y siempre presentar la cara amable del municipio a todos aquellos forasteros que necesitan y gusta visitarlo. Sim embargo, esa interacción cultural y comercial entre los habitantes del municipio y los foráneos, se ha observado que hay carencia de cultura ambiental, pues, diariamente queda en las calles un rastro enorme de desechos que ocupan el espacio público, opacan la belleza del municipio y contaminan el medio ambiente.

En el caso particular de la I.E.D. Tercera Mixta de Fundación magdalena, aun- que permanente, a través de jornadas ambientales se brinda orientación hacia el ejercicio de buenas prácticas ambientales, se ha observado que hay gran producción de residuos sólidos, además del manejo inadecuado que los estudiantes les dan a estos, desconociendo las consecuencias puede tener el trato inadecuado de los residuos sólidos para el medio ambiente y la salud de todos los que interactúan en la institución. Esta situación se hace más evidente a causa de la poca amplitud en los espacios del recreo escolar con relación a la cantidad de estudiantes que se asisten a la sede.

En consecuencia, es urgente tomar conciencia de los diferentes y grandes problemas ambientales que se generan por el uso inadecuado de los residuos sólidos, entre los que es preciso mencionar el agotamiento de los recursos energéticos y naturales. Es necesario que la educación, representada en los docentes, emprenda un camino hacia la producción de conocimiento, un conocimiento que adquiere su significancia, en cuanto surge de la necesidad e intereses de los estudiantes por intervenir situaciones de la cotidianidad que constituyen un problema social, que puede ser explorado por ellos mismos con la finalidad de sensibilizarse y lograr un ambiente escolar saludable.

Esta situación hacia la conservación del medio ambiente es, en la actualidad, un tema prioritario, pues la población mundial está viviendo las consecuencias de la falta de conciencia ambiental que hasta la actual época ha mostrado no tener el hombre, pues sus acciones para alcanzar el desarrollo y satisfacción de necesidades van en contra de su propia supervivencia y la de las generaciones futuras; fenómenos naturales, desastres naturales y extinción de las especies, son la mayor consecuencia de estas acciones, las cuales van del habitad de toda forma de vida en el planeta.

Constituyendo la Cultura Ciudadana bajo una perspectiva de vida que rige las 
actividades cotidianas creando corresponsabilidad, empoderamiento cívico y apropiación de tareas como la construcción de Ciudad y País" (Fundación Bogotá Mía, s.f.), es posible comprender que se exhorta a la participación activa de los ciudadanos, mediante acciones que garanticen el respeto por los derechos comunitarios, la sana convivencia y la protección al patrimonio cultural y natural del entorno. Entonces, si cada actor social, asume con responsabilidad su participación en los ámbitos antes mencionados, hará posible generar un impacto positivo en el ejercicio de la ciudadanía, permitiéndose satisfacer sus intereses particulares en conformidad con los del desarrollo de su sociedad.

En este orden de ideas, una clara manifestación de que la población está ejerciendo su cultura ciudadana, entre otras, está representada en el ejercicio de una conducta que propenda a la conservación del medio ambiente, teniendo en cuenta que es una acción fundamental para garantizar la calidad de vida del ser humano en el planeta, "El planeta es nuestra casa", pero es evidente que las acciones del ser humano, han cambiado las condiciones del medioambiente, poniendo en riesgo la existencia de la vida sobre la tierra, lo que es motivo de preocupación, pues son consecuencia de las inadecuadas relaciones que se han establecido entre la sociedad y el medio.

Desde esta perspectiva, es posible apelar a la función social de la educación, en cuanto a su influencia en la integración del ser humano a la sociedad y su forma de relacionarse consigo mismo, con la familia, con los demás y con su medio ambiente natural. De este modo (Montaño, 2015), que, además, expone prácticamente un consenso donde la educación tiene un papel fundamental que cumplir, mediante una transformación en la manera como actuamos en sociedad, como nos relacionamos unos con otros o como participamos para lograr los cambios que requieren los niños, niñas y jóvenes de nuestra sociedad, así como las generaciones que están por venir, reciban una formación que les permita ejercer de manera constructiva su ciudadanía (2015, p. 14).

De los anteriores planteamientos, es posible deducir que es la educación el camino para que la sociedad interiorice la cultura, pues es a través del quehacer educativo que el ser humano es capaz de construir y producir conocimientos, reorientar valores, modificar sus acciones y contribuir como sujeto individual a la transformación de la realidad del medio ambiente. Por lo que se considera que elevar el nivel de cultura ambiental de la población es una prioridad (Alaniz, 2016).

Al hilo de lo expuesto, y teniendo en cuenta la situación que da origen a la presente investigación, fortalecer la cultura ciudadana de los estudiantes a través del manejo de los residuos sólidos generados en la institución, es una acción imprescindible para favorecer el mejoramiento y protección del medio ambiente del contexto institucional proyectado al entorno comunitario de los integrantes de la comunidad educativa.

Por tales motivos Ochoa (como se citó en Sáez y Urdaneta, 2014) manifiesta que el manejo de residuos sólidos no solo comprende las actividades operativas o funcionales que se relacionan con la manipulación de estos residuos sólidos desde que son generados sino hasta la última fase que es la disposición de estos. Es necesario considerar diversos aspectos que se relacionan con el desarrollo sostenible del planeta, tal y como lo es el manejo de los residuos sólidos.

El concepto de desarrollo sostenible, propugnado en la Conferencia de las Naciones Unidas sobre el Medio Ambiente y el Desarrollo, CNUMAD 92, comprende el tema de los residuos sólidos y propicia reducir la generación de desechos, el reciclaje y reúso de todo material al máximo, y el tratamiento y disposición de los residuos en forma ambientalmente segura. Para garantizar 
el desarrollo sostenible, enunciado en el Programa 21, los gobiernos, el sector privado y las comunidades deben establecer políticas, programas y planes conjuntos donde los operadores de los servicios y la comunidad desempeñen un papel fundamental que conlleve al manejo racional de los residuos sólidos (Banco Interamericana de Desarrollo-BID, 1997).

Los residuos sólidos, hacen referencia a materiales u objetos desechados (Basuras), tras considerar que no prestan alguna a utilidad en la satisfacción de las necesidades del hombre en su cotidianidad; son el producto de materiales utilizados en la fabricación, transformación o utilización de bienes de consumo. "El problema de los residuos sólidos comenzó cuando el hombre dejó de ser nómada y se estableció en un lugar fijo. Desde entonces y periódicamente se presentan situaciones de crisis que obligan a tomar decisiones drásticas, no siempre fundadas y equivocadas" (Carega, 1997, p. 2), quiere decir entonces, que, por muchas décadas, la sociedad los ha generado indiscriminadamente, sin considerar el daño ambiental que causa su trato inadecuado, pues al ser depositados a la intemperie y sobre el suelo, generan graves problemas de contaminación, enfermedades y desequilibrio en el ecosistema natural.

Sobre el manejo adecuado de los residuos, se han realizado investigaciones a nivel internacional y nacional, las cuales constituyen un gran aporte para el desarrollo de acciones que propendan a sensibilizar a la sociedad sobre la importancia que tiene su manejo adecuado para garantizar la calidad de vida de las especies de la tierra. Al respecto, Saéz y Urdaneta (2014), en la investigación "Manejo de residuos sólidos en América Latina y el Caribe" en la que hacen una descripción de la situación actual del manejo de residuos sólidos en América Latina y el Caribe, así como las acciones y estrategias que se están empleando para mejorarlo, las autoras realizaron una revisión documental de artículos científicos y se contrastaron las realidades presentadas por los distintos autores en el manejo de residuos sólidos. El desarrollo de la investigación les permitió concluir que,

"para los países de América Latina y el Caribe la conservación del medio ambiente pasa a un segundo plano ante el número de necesidades básicas que deben cubrir, por esa razón en la mayoría de estos países los entes gubernamentales participan en la gestión de residuos sólidos realizando lo mínimo requerido para el sistema y destinando muy pocos recursos financieros para el sector. Esto trae como consecuencia que los procesos de recolección, tratamiento, aprovechamiento y disposición final de residuos sólidos sean realizados con tecnologías inadecuadas" (Saéz y Urdaneta, 2014, p. 133).

Por su parte, Rodríguez (2011) en su trabajo residuos sólidos en Colombia: su manejo es un compromiso de todos" en el que hace un análisis crítico sobre el manejo adecuado de los residuos sólidos, su influencia negativa sobre el medio ambiente, las causas del deterioro del mismo, las estrategias para minimizarlo y las posibles soluciones al problema de la falta de compromiso ciudadano en el mantenimiento y conservación del medio ambiente.

El análisis investigativo realizado por la autora, le permitió concluir que el manejo adecuado de los residuos sólidos es una necesidad imperante en nuestro mundo actual para que se puedan implementar estrategias y métodos sobre el orbe, ya que, en el mundo no se ha consolidado una cultura de conservación del medio ambiente, por lo que el Estado a través de sus representantes está llamado a reforzar e implementar métodos que permitan educar a las personas y concienticen de los riesgos que el manejo inadecuado del medio ambiente puede tener para las personas. 
"Por otro lado, es de ahí la realidad en buscar el compromiso con los valores morales, con mayor razón si se dirige a educadores y a estudiantes, ya que en gran medida está en sus manos la formación de personas, sean ellos científicos, técnicos o profesionales. Los valores morales se asumen por el ejercicio de las virtudes permanentes y universales: prudencia, justicia, fortaleza y templanza; de las que se derivan todas las virtudes que orientan los actos humanos a obrar rectamente de acuerdo con la ley moral” (Meneses, 1994, p. 94)

En este sentido, siendo el manejo inadecuado de residuos sólidos una conducta observada en la institución educativa, campo de acción donde se realiza la presente investigación, se origina la necesidad de sensibilizar e influir en la conducta del hombre para disminuir en cierto grado el impacto negativo que genera en el medio ambiente el manejo inadecuado de los residuos sólidos. De este modo, utilizando la IEP apoyada en TIC, como estrategia pedagógica, se busca contribuir al fortalecimiento de la cultura ciudadana, a través del manejo adecuado de los residuos sólidos generados en la institución.

Teniendo como línea de partida estas concepciones respecto a la producción y manejo de los residuos sólidos generados en la institución y su impacto en la conservación del medio ambiente, se propone fomentar en los estudiantes el espíritu investigador orientado al reconocimiento y solución de los problemas ambientales del entorno institucional mediante la creación de espacios de reflexión y apropiación social que propicien la participación, el intercambio de conocimientos y toma de decisiones. Así mismo dar sentido y conocimiento a la misión institucional. Elevar el nivel de cultura ambiental de la población es una prioridad, y es únicamente a través de la educación como el individuo interioriza la cultura, y es capaz de construir y producir conocimientos, reorien- tar sus valores, modificar sus acciones y contribuir como sujeto individual a la transformación de la realidad del medio ambiente (Alaniz, 2016).

Para ello, se propone el diseño y desarrollo de estrategias que permitan contribuir al fortalecimiento de la cultura ciudadana a través del manejo adecuado de los residuos sólidos apoyados en la investigación como estrategia pedagógica (IEP) mediada por las TIC, promoviendo el emprendimiento, la innovación y el aprendizaje. Así mismo, se cuenta con el apoyo de todos los entes de la gestión escolar y existen los espacios y recursos para ser implementada.

Razón por la cual, se promovió un proceso investigativo para contribuir al fortalecimiento de la cultura ciudadana de los estudiantes a través del manejo de los residuos sólidos generados en la institución. Este proceso partió de un diagnóstico veraz que caracterizó las dinámicas institucionales respecto al manejo de los residuos sólidos; se desarrolló a través de un proceso de indagación, que permitió conocer las concepciones y hábitos de los estudiantes con respecto al manejo de los residuos sólidos generados en la institución, es importante aclarar no se dio como una simple actividad de aplicación de instrumentos, sino, como la apropiación de un espacio social en el que los estudiantes pudieron compartir y reflexionar sobre sus conocimientos, acciones, creencias y actitudes respecto al manejo de los residuos sólidos.

Así mismo, tuvieron la posibilidad de indagar para construir conocimientos contextualizados sobre el tema planteado, fomentando en ellos el espíritu investigador orientado al reconocimiento y solución de los problemas ambientales producidos por manejo inadecuado de los residuos sólidos generados en el entorno institucional. La caracterización de las dinámicas institucionales respecto a ma- 
nejo de los residuos sólidos, siguiendo las orientaciones del programa Ciclón y desde la naturaleza de cada disciplina, instó a los docentes a desarrollar un proceso de integración curricular, para lo cual, se diseñaron estrategias pedagógicas transversales que se implementaron a través de actividades de indagación para promover espacios de apropiación que propiciaran en los estudiantes la producción de saberes, la participación, el intercambio de conocimientos.

Desde los planteamientos anteriores, es claro que a nivel institucional se generó una situación de movilización de todos los actores del proceso educativo, y aunque no es fácil salir de la zona de confort en la que se han posicionado muchos docentes en el ejercicio de sus prácticas pedagógicas y asumir los cambios que implica la apropiación curricular de la IEP, a partir del proceso de formación, el intercambio de experiencias, conocimientos y la implementación de estrategias, se instaló la capacidad para hacer de la IEP una opción para trasformar las prácticas educativas tradicionales.
Así pues, y en concordancia con tales políticas, es importante en un diseño que instrumenten programas que fomenten, entre otros, la prevención de la contaminación y la reducción de residuos en la fuente. Asimismo, se realizará la evaluación periódica de las actividades para determinar su desempeño ambiental respecto de las metas previamente establecidas y se buscará la mejora continua.

Al mismo tiempo, se fomentará que los proveedores y contratistas del sistema adopten los principios ambientales que sean prudentes y convenientes, seleccionando a aquéllos que se desempeñen mejor. A la vez, se difundirán las actividades de protección ambiental que se desarrollen, para hacerlas del conocimiento de todos los involucrados en el manejo integral de los residuos sólidos y del público en general. Será responsabilidad de los trabajadores conocer, entender y contribuir a la puesta en marcha de la política ambiental, en tanto que los administradores del sistema de aseo urbano deberán asegurar que la selección, diseño y operación de las instalaciones sean ambientalmente adecuadas.

TABLA 1

Planteamientos de una politica municipal de manejo ambiental de residuos

\begin{tabular}{|c|c|c|c|}
\hline Propósitos & Enfoque & Compromisos & Elementos \\
\hline $\begin{array}{l}\text { Esta política ambiental } \\
\text { ha sido desarrollada } \\
\text { para ayudar al } \\
\text { personal involucrado en } \\
\text { el sistema municipal de } \\
\text { aseo urbano, así como } \\
\text { a los proveedores y } \\
\text { contratistas que } \\
\text { participan en él, } \\
\text { a desarrollar sus } \\
\text { actividades en forma } \\
\text { ambientalmente } \\
\text { adecuada. }\end{array}$ & $\begin{array}{l}\text { La Política aplica a } \\
\text { todos los empleados, } \\
\text { proveedores y } \\
\text { contratistas, así } \\
\text { como a todas las } \\
\text { operaciones que } \\
\text { realiza el sector de } \\
\text { aseo urbano, y a las } \\
\text { actividades y } \\
\text { procesos que } \\
\text { involucra el manejo } \\
\text { integral de los } \\
\text { residuos sólidos } \\
\text { municipales. }\end{array}$ & $\begin{array}{l}\text { A través de esta } \\
\text { política, el sistema } \\
\text { municipal de } \\
\text { aseo urbano, se } \\
\text { compromete } \\
\text { a contribuir } \\
\text { al desarrollo } \\
\text { sustentable, } \\
\text { orientado a hacer } \\
\text { compatible el } \\
\text { crecimiento } \\
\text { económico y la } \\
\text { protección al } \\
\text { ambiente }\end{array}$ & $\begin{array}{l}\text { En la operación del sistema se } \\
\text { considera básica la prevención y } \\
\text { reducción de la } \\
\text { contaminación, aunada } \\
\text { a la optimización del } \\
\text { aprovechamiento de los recursos, } \\
\text { incluyendo las sustancias, } \\
\text { agua y energía; para lo cual se } \\
\text { seguirán lineamientos } \\
\text { ambientales en las decisiones } \\
\text { que se adopten, incluyendo las } \\
\text { relativas a proyectos } \\
\text { de expansión, mantenimiento, } \\
\text { renovación y adquisición. }\end{array}$ \\
\hline
\end{tabular}

Fuente: elaboración propia. 


\section{Metodología}

Se realizó un estudio de tipo cualitativo, utilizando el modelo de investigación acción, desde un diseño descriptivo, exploratorio. La investigación cualitativa busca conocer e interpretar la realidad de los participantes a través de sus propias experiencias, entregando una información subjetiva del fenómeno de estudio. (Hernández, Fernández, y Baptista, 2010). Por su parte el modelo de investigación acción plantea una producción de conocimiento basado en la reflexión de los participantes, teniendo en cuenta la participación activa de maestros en el proceso de enseñanza y acompañamiento tecnológico, guiando al estudiante en su proceso de investigación. Generando nuevo saber y conocimiento sobre una realidad determinada en un proceso de aprendizaje colaborativo.

Según Cruz (2016) los estudios con un diseño descriptivo están encaminados a especificar las características de la población sujeto de estudio o los fenómenos que sean sometidos a análisis. Bajo un estudio de tipo exploratorio, debido a que no se han realizado estudios previos en la institución (Hernández, Fernández, y Baptista, 2010). La población sujeto de estudio estuvo constituida por sesenta (60) estudiantes de preescolar y educación básica ciclo secundaria. La participación de cada uno de actores involucrados se dio como una práctica de intercambio de conocimientos, experiencias y capacidades que contribuyeron al logro de los objetivos propuestos, y por consiguiente dar respuesta, diseñar estrategias e implementar acciones que permiten la solución al problema de investigación planteado.

Se emplearon técnicas que permitieron sistematizar y conocer de manera descriptiva la variable a investigar tales como: la observación participante y como instrumento el diario de campo, de igual forma, se utilizó la encuesta. Adicional se hicieron registros fotográficos, historias de vida y entrevistas con el fin de facilitar la descripción de los procesos desarrollados durante la investigación e implementación de la propuesta.

Para conocer las concepciones y hábitos de los estudiantes con respecto al manejo de los residuos sólidos, se aplicó un cuestionario, diseñado y validado por un equipo interdisciplinar de la institución. Este instrumento se construyó en forma digital on-line, elaborado en Google Form y el link generado (https://goo.gl/forms/GRpwBR6RuXKl0Z0c2) compartido con los estudiantes para su diligenciamiento en un espacio de apropiación, que les permitirá reflexionar en torno a sus conocimientos, acciones y actitudes sobre el tema abordado. El cuestionario se estructuró en cuatro sesiones que responden a los niveles de competencia a desarrollar en los estudiantes durante el proceso investigativo sobre el manejo de los residuos sólidos generados en la institución:

Sesión I. Conocimientos sobre preservación del medio ambiente.

Sesión II. Conocimientos sobre manejo de residuos sólidos.

Sesión III. Manejo de los residuos sólidos generados en la institución.

Sesión IV. Valores y creencias respecto a la conservación del medio ambiente y manejo de residuos sólidos.

Para el registro fotográfico se parte de la premisa de que, la fotografía permite observar, analizar y teorizar la realidad social. Más específicamente, la imagen como dato ayuda a contextualizar lo observado y posibilita profundizar sobre aspectos menos visibles en otros modos de registro de lo observado" (Bonetto, 2016, p. 71), los registros fotográficos se utilizaron para evidenciar de forma concreta, la realidad cotidiana sobre el trato que se da a los residuos sólidos que se generan en la institución durante las dinámicas cotidianos de interacción entre los integrantes de la co- 
munidad educativa. Estos registros serán tomados por los mismos estudiantes, de tal forma que sean ellos quienes perciban el grado de responsabilidad de cada uno en el origen de la situación estudiada.

En el caso del Diario de Campo permitió al equipo investigador, sistematizar las acciones investigativas desarrolladas en todos las fases y etapas de implementación de la ruta metodológica de la IEP. A través de este instrumento, fue posible llevar los registros y anotaciones de lo acontecido en cada una de las actividades desarrolladas para cumplir con los objetivos y de esta forma dar respuesta al problema de investigación. El formato del instrumento fue diseñado y validado por el equipo interdisciplinar de la institución, su diseño permitió recoger datos relacionados con los propósitos de las actividades, los participantes, las descripciones de lugar y tiempo, las observaciones, interpretaciones y evidencias de las actividades desarrolladas, este registro de información, permitió al equipo reflexionar sobre lo hallazgos descubiertos en cada etapa, y de esta forma construir los saberes y conocimientos necesarios para comprender el problema de investigación.

La historia de vida es la técnica que permite al investigador conocer un suceso o fenómeno desde la perspectiva que quien, en las dinámicas de la cotidianidad, lo vive directamente, es comprensible entonces, concebirlo como, "El relato de un narrador sobre su existencia a través del tiempo, intentando reconstituir los acontecimientos que vivió y transmitir la experiencia que adquirió" Pereira de Queiroz (1991, citado por Veras, 2010, p.144). En este sentido, para el desarrollo de la investigación, la historia de vida se ha implementado para conocer opciones para el manejo de los residuos sólidos, no desde los referentes teóricos, sino de viva voz de un personaje, quien ha hecho del reciclaje una opción de vida para él y su familia.
La entrevista se utilizó con la finalidad de conocer las posibilidades y beneficios que se pueden obtener con el trato adecuado de los residuos sólidos. La entrevista fue realizada a un habitante del municipio de Fundación, quien se dedica formalmente al reciclaje de residuos sólidos generados en el municipio. La estructura de la entrevista fue diseñada por el equipo investigador, pero fue aplicada por los estudiantes en formato de vídeo (Ver Anexo No. 3), con la finalidad de desarrollarán un proceso de indagación que les permitiera a los estudiantes construir conocimientos contextualizados sobre el manejo de los residuos sólidos, fomentando en ellos espíritu investigador orientado al reconocimiento y solución de los problemas ambientales producidos por manejo inadecuado de los residuos sólidos generados en el entorno institucional.

\section{Resultados}

La implementación de la trayectoria de indagación permite al equipo investigador conocer a fondo la temática y el problema a investigar. A continuación, se describen los resultados más significativos obtenidos en cada uno de las sesiones en las que se desarrolló la trayectoria de investigación.

Se realizaron las actividades para identificar las concepciones y hábitos de los estudiantes con respecto al manejo de los residuos sólidos generados en la institución. La aplicación de los instrumentos como la encuesta y la observación directa (Registros fotográficos) permitió determinar que, en cuanto a los cocimientos de los estudiantes sobre preservación ambiental y manejo de residuos sólidos, la mayoría de ellos tiene conocimiento de que algunas acciones causan contaminación ambiental y el beneficio de otras acciones como reciclar, representa beneficios para el medio ambiente. 
Así mismo, los estudiantes, manifiestan identificar diferentes tipos de residuos sólidos y cuáles de ellos se generan en la institución, conocen la función y la forma de depositarlos residuos en los puntos ecológicos y manifiestan conocer entidades municipales encargadas de la problemática ambiental y el manejo que se da a los residuos generados en el Municipio; este nivel de conocimiento que manifiestan los estudiantes sobre el tema, es comprensible ya que, la temática es permanentemente trabajada a nivel institucional. Sin embargo, un gran porcentaje no sabe de la existencia de una entidad municipal que se encargue de la problemática ambiental, además, en cuanto al manejo de los residuos, no tienen claridad en cuáles de ellos se pueden Reciclar, Reutilizar y Reducir su consumo. Así mismo, un número elevado de ellos, no tiene conocimiento sobre la existencia de una entidad municipal que se encargue de la problemática ambiental.

En cuanto a las acciones de los estudiantes respecto al manejo de los residuos sólidos, la mayoría de ellos manifiesta hacer manejo adecuado de residuos sólidos que genera con el consumo en la institución, no obstante, los registros fotográficos dejan evidencia de que un porcentaje muy significativo de ellos, no ejerce un manejo adecuado sobre ellos, ya que, diariamente y en diferentes momentos de la jornada escolar, se observan estos residuos en los pisos de diferentes áreas institucionales a las que ellos tienen acceso.

En cuanto a los valores y actitudes de los estudiantes sobre la conservación ambiental y manejo de residuos sólidos, casi la totalidad de los estudiantes encuestados reconocen su responsabilidad en la conservación del medio ambiente, saben que sus acciones pueden favorecer o no su conservación y si en su interacción diaria observan a un compañero dar un manejo inadecuado a los residuos sólidos, están en disposición de llamar su atención. No obstante, es significativo que algunos estudiantes, no asuman esa posición.
También se realizaron las actividades de contextualización para fomentar en los estudiantes el espíritu investigador orientado al reconocimiento y solución de los problemas ambientales producidos por manejo inadecuado de los residuos sólidos generados en el entorno institucional. Los resultados más significativos obtenidos durante la implementación de las actividades fueron:

La visita a uno de los puntos o lugares de reciclaje que funcionan en el municipio fue posible enterar a la comunidad educativa de la existencia de una empresa municipal, legalmente constituida que vela por la conservación del medio ambiente "FUNDASEO". La empresa de acuerdo a lo manifestado por el administrador, hace aproximadamente seis años se dedica al aprovechamiento de los residuos sólidos, ya que ellos reciclan y reutilizan los productos que recolectan: cartón, papel limpio y sucio, plástico, botellas, chatarra, entre otros. De su voz fue posible conocer el valor al que una persona puede venderles a ellos cada producto y el aprovechamiento específico de cada residuo. Así mismo, el administrador abrió la posibilidad a los estudiantes de cuidar sus cuadernos para que al finalizar el año escolar pueda venderlos y utilizar el dinero para comprar nuevos cuadernos, lo cual representa una alternativa eficaz para reducir la cantidad de papel que diariamente se genera en la institución.

La historia de vida de uno de los retomadores ambientales de la empresa FUNDASEO, "Gustavo Plata" reconocido en el municipio por su historia de superación y preocupación por la conservación ambiental del municipio, pues a través de su oficio a logrado cursar sus estudios tecnológicos para la conservación del medio ambiente, realizar investigaciones sobre el uso de residuos orgánicos como abono y crear un vivero de plantas ornamentales y medicinales para promover la conservación ambiental y para su sustento económico; hace parte de la RED Latinoamérica de recicladores y por su labor, en el año 2006 se le fue otorgado el reconocimiento 
como el mejor reciclador de Latinoamérica. Él manifiesta que ve en la problemática de la producción de residuos sólidos una opción económica para la supervivencia, además de la satisfacción de ayudar a la conservación del medio ambiente.

\section{Conclusión}

Al integrar la IEP al currículo, se diseñaron e implementaron las estrategias didácticas transversales en torno al manejo de los residuos sólidos generados en la institución, pero desde la naturaleza de cada disciplina. La implementación de la investigación como estrategia pedagógica, fomenta una cultura de ciencia y tecnología mediante el apoyo a investigaciones sugeridas y desarrolladas por los niños, niñas y jóvenes de las diferentes regiones del país (Manjarréz, 2007).

La iniciación y consolidación de procesos de investigación, a partir de las preguntas de los niños y jóvenes para resignificar el trabajo escolar y su vida cotidiana. Para lograrlo, se motiva a los niños, niñas y jóvenes para que, en compañía de adultos, los estudiantes puedan diseñar y desarrollar proyectos de investigación en todas las áreas del saber, de tal modo que se familiaricen con los métodos y el lenguaje de la ciencia, y se reconozcan como personas capaces de producir conocimiento e incidir en la solución de problemas de su región y del país (2007).

A partir del proceso de investigación vivenciado se consolidó la una propuesta para integrar la I.E.P. apoyada en TIC al currículo, que, siguiendo su ruta metodológica, se dio como una actividad de 'negociación cultural', 'diálogo de saberes' y construcción de aprendizajes' en torno a la producción y manejo de los residuos sólidos generados en la institución. De este modo se generó la posibilidad de posibilidad de propiciar en los estudiantes la construcción de un aprendizaje situado, colaborativo, problematizador y por indagación, ya que, se toma la pregunta como punto de partida en atención a los saberes previos que manejan los estudiantes, se propicia un dialogo de saberes, en la medida en que se rompe el dualismo ellos-los otros. Se relaciona su saber con los saberes de los otros (sociedad), confrontado a los saberes existentes en la sociedad y se utilizan las TIC en reconocimiento a que las mediaciones son parte de su mundo y de la manera como construye (Recursos y medios) conocimiento.

Al finalizar la trayectoria de indagación investigación y a partir de los resultados obtenidos, después de vivenciar el proceso de investigación es posible afirmar que:

1. Aunque los estudiantes posean sus conocimientos en torno a la importancia de la conservación ambiental y el manejo adecuado de los residuos sólidos, esto no suficiente, se necesita el desarrollo de actividades que para ellos resulten significativas para que puedan apropiarse y ejercer competentemente los saberes construidos.

2. La implementación de estrategias pedagógicas transversales basadas en la indagación fomenta en los estudiantes el espíritu investigador, ya que, vivenciar el proceso posibilita que ellos ejerzan un rol protagónico como actores proactivos en la construcción de sus aprendizajes y de este modo pueden hacer un real reconocimiento de los problemas y ambientales que produce el trato inadecuado de los residuos sólidos en su entorno comunitario e institucional.

3. Los espacios de apropiación social, en los que los estudiantes participan dando a conocer sus experiencias, los aprendizajes construidos e intercambiando sus saberes con otros, les permite reflexionar sobre sus actitudes y aptitudes en torno a la conservación del medio ambiente, y por consiguiente los insta a tomar decisiones trascendentales su proceso de formación y educación.

4. Las comunidades virtuales de práctica y aprendizaje promueven el aprendizaje autónomo en los estudiantes, pues estas se construyen con los aportes que él puede hacer desde su experiencia en torno a la temática propuesta. 
En este sentido, se ha iniciado el camino para fortalecer la cultura ciudadana de los estudiantes a través del manejo de los residuos sólidos generados en la institución, y aunque es claro que no es se pueden obtener resultados inmediatos, se ha evidencia en los espacios institucionales que se está dando el aprendizaje en ellos.

\section{Referencias}

Alaniz, E. (2016). La cultura ciudadana, protectora del medio ambiente. Recuperado de http://news.agrofy.com.ar/ noticia/160791/cultura-ciudadanaprotectora-medio-ambiente

Arrieta, M. y Rojas, A. (2002). Análisis de las garantías del proceso de alimentos y su eficacia dentro del proceso. [Tesis de pregrado]. Universidad del Norte, Barranquilla, Colombia.

Banco Interamericana de Desarrollo- BID. (1997). Diagnóstico de la situación del manejo de residuos sólidos municipales en América Latina y el Caribe. Recuperado de https://publications.iadb.org/handle/11 319/4768\#sthash.0EyYP1KR.dpuf

Bonetto, M. (2016). El uso de la Fotografía en la investigación social. Revista Latinoamericana de Metodología de la Investigación Social. 11(6). 71-83. Recuperado de http://www.relmis.com.ar/ojs/index.php/ relmis/article/view/151/230

Borda, M. (2013). El proceso de investigación: visión general de su desarrollo. Barranquilla: Universidad del Norte.

Cabello-Eras, J. (2016). Acercamiento a la producción más limpia como estrategia de gestión ambiental. Ijmsor, 1(1), 4-7. Recuperado de http://ijmsoridi.com/index.php/ ijmsor/article/view/71

Carega, J. (1997). Folleto sobre el reciclaje. México, D.F.: INARE.

Cortés-Peña, O. (2016). Sustainable development in synergistic relationship with proambient behavior and fair trade. Ijmsor, 1(1), 54-58. Retrieved from http://ijmsoridi.com/index.php/ijmsor/article/view/78
Cruz, E. (2016). El ciclo de protesta 2010-2016 en Colombia. Una explicación. Jurídicas CUC, 12 (1), 31-62. DOI: http://dx.doi. org/10.17981/juridcuc.12.1.2016.3

Fundación Bogotá Mía. (s.). Cultura Ciudadana. Recuperado de http://www.fundacionbogotamia.org/culturaciudadana.html

Manjarréz, M. (2007). La investigación como estrategia pedagógica del programa Ondas de Colciencias. En, UNESCO. X Reunión de la Red de Popularización de la Ciencia y la Tecnología en América Latina y el Caribe - RED POP y IV Taller Ciencia, Comunicación y Sociedad. San José, Costa Rica.

Meneses, O. (1994). La profesión de Educar. [Tesis]. Universidad de la Sabana. Bogotá, D.C. Recuperado de https://goo.gl/jCtXgi

Meza, A., Arrieta, M. y Noli S. (2018). Análisis de la conciliación extrajudicial civil en la Costa Atlántica colombiana. Jurídicas CUC, 14(1). 187-210. DOI: http://dx.doi. org/10.17981/juridcuc.14.1.2018.9

Montaño, E. (2015). Estrategia Educativa Apoyada en Tic para la Formación en Ciudadanía y Convivencia en Estudiantes de Secundaria de Grado Décimo y Undécimo. [Tesis]. Universidad Pedagógica Nacional. Bogotá, D.C.

Nieves, J.ySalazar,F.(2016).e-Supply chain management para una empresa de tecnología. Ijmsor, 1(1),31-34. Recuperado de http:// ijmsoridi.com/index.php/ijmsor/article/ view/74

Rodríguez. S. (2011). Residuos Sólidos en Colombia: Su manejo es un compromiso de todos. L'spirit ingenieux, 2(1). 91-96. Recuperado de http://revistas.ustatunja.edu.co/ index.php/lingenieux/issue/view/9

Saéz, A. y Urdaneta, J. (2014). Manejo de residuos sólidos en América Latina y el Caribe. Omnia, 20(3), 121-135. Recuperado de http:// www.redalyc.org/pdf/737/73737091009.pdf

Sagastume, A. y Cabello, J. (2017). La educación superior y una producción más limpia. Ijmsor, 2(1), 4-8. Recuperado de http:// ijmsoridi.com/index.php/ijmsor/article/ view/79 